02,12

\title{
Динамическая восприимчивость нанокомпозита пористое стекло/Ga-In-Sn в области сверхпроводимости
}

\author{
() О.Д. Шевцова ${ }^{1}$, М.В. Лихолетова ${ }^{1}$, Е.В. Чарная ${ }^{1}$, Е.В. Шевченко ${ }^{1}$, Ю.А. Кумзеров ${ }^{2}$, А.В. Фокин ${ }^{2}$ \\ ${ }^{1}$ Санкт-Петербургский государственный университет, \\ Санкт-Петербург, Россия \\ ${ }^{2}$ Физико-технический институт им. А.Ф. Иофффе РАН, \\ Санкт-Петербург, Россия \\ E-mail: ezhi9327@gmail.com
}

Поступила в Редакцию 30 августа 2021 г.

В окончательной редакции 30 августа 2021 г.

Принята к публикации 3 сентября 2021 г.

В последнее время возрос интерес к исследованиям сплавов галлия в связи с их возможными применениями для самовосстанавливающихся сверхпроводящих контактов и проводов. Особое внимание обращается на сверхпроводящие свойства наноструктурированных сплавов. В настоящей работе проведены исследования динамической $(a c)$ восприимчивости нанокомпозита пористое стекло/Ga- $\mathrm{In}-\mathrm{Sn}$ в температурном диапазоне $1.9-8 \mathrm{~K}$ и полях смещения до $5 \mathrm{~T}$. Выявлено два сверхпроводящих перехода с температурами 5.6 и $3.1 \mathrm{~K}$. Построены фазовые диаграммы. Продемонстрированы участки положительной кривизны критических линий, интерпретируемые в рамках модели, учитывающей эффект близости. По смещению максимумов мнимой части восприимчивости при изменении частоты переменного поля определены активационные барьеры для подвижности вихрей. Показано наличие излома на полевой зависимости активационных барьеров.

Ключевые слова: тройной сплав $\mathrm{Ga}-\mathrm{In}-\mathrm{Sn}$, нанокомпозит, сверхпроводимость, магнитометрия.

DOI: 10.21883/FTT.2022.01.51829.195

\section{1. Введение}

В настоящее время большой интерес вызывают исследования сверхпроводимости в наноструктурированных системах. Достигнутый в этом направлении прогресс привел к существенным успехам как в прикладной области, так и в фундаментальной физике [1,2]. Значительные усилия концентрируются на проблеме создания наносверхпроводников с различной морфологией, что позволяет влиять на сверхпроводящие свойства. Одна из возможностей изменять морфологию сверхпроводников состоит в использовании твердых мезопористых матриц, в которые различными способами вводятся сверхпроводящие материалы $[3,4]$. К таким матрицам относятся, например, силикатные пористые стекла, цеолиты, асбесты и опалы. Использование пористых матриц с разной геометрией сетки пор позволяет варьировать форму сверхпроводящих включений и их взаимное расположение. При этом характерные размеры сверхпроводников за счет наноконфайнмента могут сокращаться ниже длины когерентности и глубины проникновения магнитного поля в соответствующих объемных материалах. В условиях наноконфайнмента реализуется дендритная форма сверхпроводящих включений [5-8], что является сложной задачей для других технологий из-за проблем с хрупкостью.

Недавние исследования нанокомпозитов на основе пористых матриц, заполненных сверхпроводящими металлами, выявили ряд общих закономерностей на фоне индивидуальных различий, связанных с размером пор, связностью сверхпроводящей фазы и морфологией сверхпроводящих включений [5-12]. Наноконфайнмент приводит к изменению рода сверхпроводимости за счет уменьшения длины когерентности, а также к возрастанию значений верхнего критического поля и критического тока. Наноконфайнмент может усиливать склонность металлов к полиморфизму, что сопровождается появлением нескольких температур сверхпроводящего перехода для галлия в опалах и пористых стеклах. С другой стороны, наличие в нанокомпозите сильных и слабых джозефсоновских контактов также может отвечать за ступенчатый характер сверхпроводящего перехода. Для металлов в одинаковых опаловых матрицах наблюдались близкие фазовые диаграммы. Во многих случаях на температурных зависимостях критических полей имелись области с положительной и отрицательной кривизной. Исследования динамической восприимчивости выявили сложную зависимость энергии активации движения сверхпроводящих вихрей от постоянного магнитного поля. Более сложная конфигурация сверхпроводящей фазы в эвтектических металлических сплавах может приводить к новым эффектам по сравнению с нанокомпозитами с включениями чистых металлов. Однако такие исследования были проведены только для бинарного эвтектического сплава $\mathrm{Ga}-\mathrm{Ag}$ [13].

В настоящей работе представлены результаты исследования сверхпроводимости нанокомпозита силикатное пористое стекло/тройной сплав галлия, индия и олова. Проводились измерения динамической $(a c)$ намагничен- 
ности при приложении различных смещающих магнитных полей. Сплавы галлия, в особенности бинарный сплав галлия с индием и тройной сплав галлия с индием и оловом, вызывают большой интерес в связи с перспективами использования в различных современных областях, таких как робототехника и микроэлектроника. Тройной сплав $\mathrm{Ga}-\mathrm{In}-\mathrm{Sn}$ и аналогичный сплав с небольшой добавкой других элементов (галинстан) рассматриваются как перспективные материалы для сверхпроводящих контактов и проводов $[14,15]$ благодаря возможности восстанавливаться после нагрева до комнатной температуры. Сверхпроводимость в объемном сплаве $\mathrm{Ga}-\mathrm{In}-\mathrm{Sn}$ с составом, близким к эвтектической точке, исследовалась в работе [16]. Сверхпроводящие свойства нанокапель $\mathrm{Ga}-\mathrm{In}-\mathrm{Sn}$ со средним диаметром $110 \mathrm{~nm}$ и различным соотношением компонент изучались в работе [17]. Переход в сверхпроводящее состояние объемного сплава эвтектической концентрации наблюдался при $6 \mathrm{~K}$, тогда как для нанокапель максимальная температура сверхпроводящего перехода достигала $6.6 \mathrm{~K}$.

\section{2. Эксперимент}

Пористое стекло, используемое в качестве матрицы, было получено из натриево-боратного силикатного стекла путем ликвации и выщелачивания. Средний размер пор по данным азотной порометрии равнялся $7 \mathrm{~nm}$. Тройной сплав $\mathrm{Ga}-\mathrm{In}-\mathrm{Sn}$ вводился в поры при комнатной температуре под давлением до $10 \mathrm{kBar}$. Состав сплава был близок к эвтектическому: 77.2 at.\% Ga, 14.4 at.\% In, и 8.4 at.\% Sn [18]. Температура плавления объемного сплава в эвтектической точке равна $283.7 \mathrm{~K}$ [18], так что при комнатной температуре сплав находится в жидком состоянии. Заполнение пор составляло около $80 \%$ по оценкам веса пористого стекла до и после заполнения. Исследуемый образец вырезался из заполненной матрицы и тщательно очищался от объемного сплава на поверхности. Вес образца равнялся $24.25 \mathrm{mg}$.

Комплексная динамическая $(a c)$ намагниченность измерялась на установке PPMS-9+EverCool-II производства Quantum Design. Температурные зависимости намагниченности были получены в режиме охлаждения при приложении различных внешних магнитных полей на различных частотах и с различными амплитудами переменного поля. Температура изменялась от 1.9 до $8 \mathrm{~K}$.

\section{3. Результаты и обсуждение}

Температурные зависимости вещественной $\chi^{\prime}$ и мнимой $\chi^{\prime \prime}$ частей $a c$ восприимчивости, полученные при приложении различных смещающий магнитных полей, представлены на рис. 1. Измерения проводились на частоте $5.01 \mathrm{kHz}$. Амплитуда переменного поля составляла 2 Ое. На кривых восприимчивости в магнитных полях

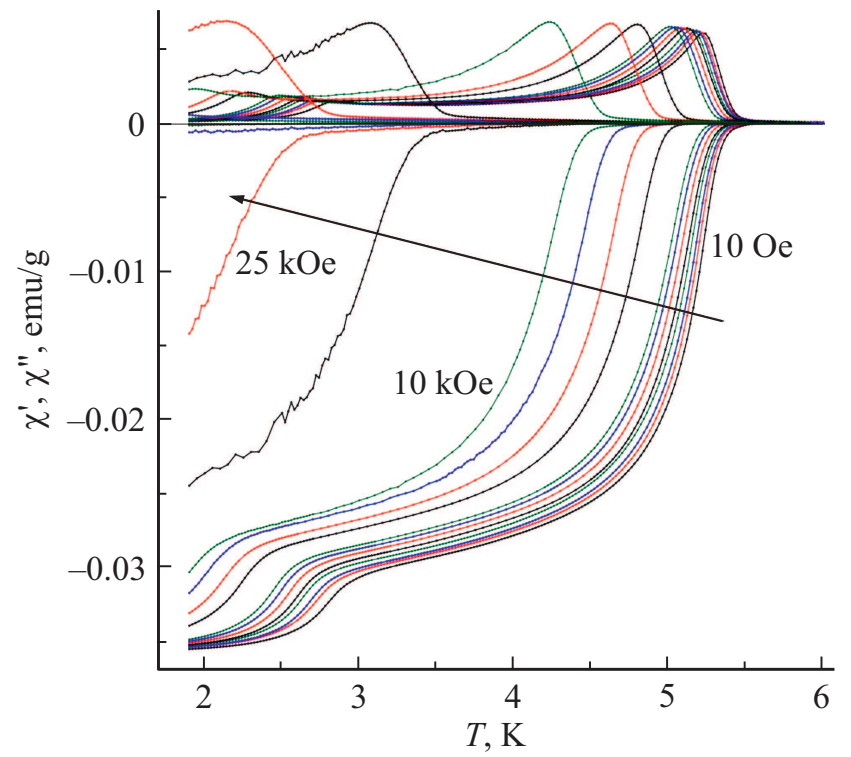

Рис. 1. Температурные зависимости вещественной (внизу) и мнимой (вверху) частей восприимчивости $\chi$, измеренные в смещающих полях $10,50,100,200,300,500,750$ Ое и 1,3 , $5,7.5,10,20,25,30,40 \mathrm{kOе.} \mathrm{Стрелка} \mathrm{показывает} \mathrm{увеличение}$ поля. Для наглядности около трех зависимостей указаны величины полей.

до $10 \mathrm{kOe}$ видны две ступени для $\chi^{\prime}$ и два пика для $\chi^{\prime \prime}$. Обе ступени на вещественной части восприимчивости размыты даже в низком поле 10 Ое. С ростом смещающего поля ступени и пики сдвигаются к низким температурам, так что в полях, больших $10 \mathrm{kOe}$, наблюдается только одна ступень на кривой $\chi^{\prime}(T)$. Для характеристики сверхпроводимости в исследуемом нанокомпозите мы ввели в рассмотрение четыре температуры, используя данные на рис. 1 . Температура $T_{c}$ характеризует установление сверхпроводящего состояния. Мы рассчитывали ее как температуру, при которой первая производная вещественной части восприимчивости по температуре равнялась $2 \%$ от своего максимального значения. Для характеристики второй, низкотемпературной, ступени на вещественной части восприимчивости мы ввели температуру $T_{c}^{\prime}$, при которой первая производная возрастала ниже локального минимума, находящегося непосредственно выше второй ступени, на $0.5 \%$. Температуры $T_{p}$ и $T_{p}^{\prime}$ показывают положения максимумов мнимой части восприимчивости для первого (высокотемпературного) и второго (низкотемпературного) пиков соответственно. Зависимости этих четырех температур от смещающего поля показаны на фазовой диаграмме на рис. 2.

Согласно рис. 1 и 2 температура перехода в сверхпроводящее состояние для наноструктурированного сплава $\mathrm{Ga}-\mathrm{In}-\mathrm{Sn}$ эвтектического состава равна $T_{c}(0)=5.6 \mathrm{~K}$. Эта температура значительно выше температур сверхпроводимости для трех компонент сплава $(1.08 \mathrm{~K}$ для $\alpha$ - Ga, $3.41 \mathrm{~K}$ для In и $3.73 \mathrm{~K}$ для $\mathrm{Sn})$. Она отличается от критической температуры около $6 \mathrm{~K}$, найденной для 


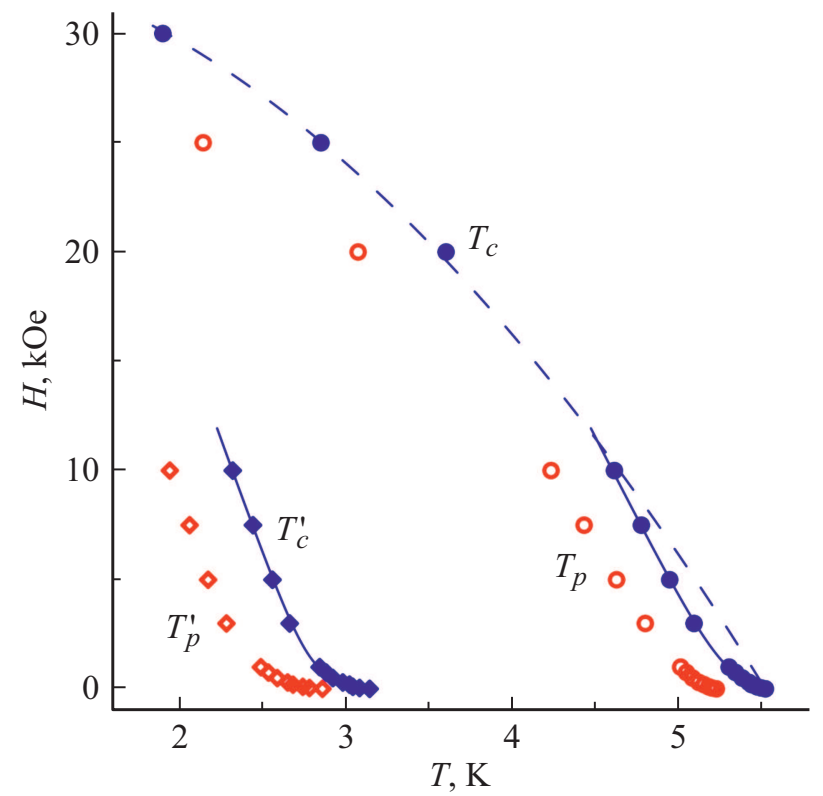

Рис. 2. Фазовая диаграмма для первого (кружки) и второго (ромбы) сверхпроводящих переходов. Темные символы температуры переходов, светлые символы - температуры максимумов мнимой части восприимчивости. Штриховая линия - теоретическая зависимость для модели двух жидкостей. Сплошные линии - теоретические кривые для модели, разработанной в работе [23].

объемного тройного сплава с соотношением компонент $78: 15: 7$ в работе [16]. Температура сверхпроводимости в исследуемом нами нанокомпозите также ниже, чем максимальная температура перехода $6.6 \mathrm{~K}$, найденная для объемного тройного сплава $\mathrm{Ga}-\mathrm{In}-\mathrm{Sn}$ и нанокапель определенного состава [17]. Недавние исследования кристаллической фазы в объемном сплаве $\mathrm{Ga}-\mathrm{In}-\mathrm{Sn}[16]$ продемонстрировали формирование интерметаллических соединений $\mathrm{In}_{3} \mathrm{Sn}$ и $\mathrm{InSn}_{4}$. Такие соединения образуются также при кристаллизации сплава In-Sn $[19,20]$. Соединение $\operatorname{In}_{3} \mathrm{Sn}$ переходит в сверхпроводящее состояние при температурах выше $5 \mathrm{~K}$ [20]. Таким образом, следует предположить, что найденная нами температура перехода в сверхпроводящее состояние связана с присутствием в нанокомпозите интерметаллического соединения $\operatorname{In}_{3} \mathrm{Sn}$, образовавшегося при кристаллизации тройного сплава $\mathrm{Ga}-\mathrm{In}-\mathrm{Sn}$ эвтектического состава. Низкотемпературная ступенька на вещественной части намагниченности характеризуется температурой $3.1 \mathrm{~K}$ в нулевом магнитном поле. Эта температура близка к температуре сверхпроводимости объемного индия. Можно предположить, что вторая ступенька на зависимости $\chi^{\prime}(T)$ обусловлена формированием кристаллического твердого раствора индия с небольшим количеством олова и галлия. Отметим, что в работах $[16,17]$ не сообщалось о втором сверхпроводящем переходе в тройном сплаве $\mathrm{Ga}-\mathrm{In}-\mathrm{Sn}$.
Зависимости верхнего критического поля от температуры $H_{c 2}(T)$ на фазовой диаграмме (рис. 2) имеют вид, отличающийся от традиционных зависимостей WHH [21] или модели двух жидкостей [22], наблюдаемых для сверхпроводников второго рода. Для первого перехода кривизна является положительной при низких полях и становится отрицательной выше $10 \mathrm{kOe}$. Для второго перехода кривизна остается положительной во всем температурном диапазоне измерений. Следует отметить, что зависимости $T_{p}(H)$ также имеют участки аномальной кривизны и следуют кривым $H_{c 2}(T)$.

Участок фазовой диаграммы для первого перехода в области отрицательной кривизны допускает интерпретацию в рамках модели двух жидкостей [22]:

$$
H_{c 2}(T)=H_{c 2}(0)\left[1-\left(\frac{T}{T_{c}}\right)^{2}\right],
$$

при $H_{c 2}(0)=3.38 \mathrm{kOe}$. Полученное значение верхнего критического поля при нулевой температуре близко к оценке для объемного сплава $\mathrm{Ga}-\mathrm{In}-\mathrm{Sn}$ в работе [16]. Согласно теории Ландау верхнее критическое поле связано с длиной когерентности

$$
\xi(0)=\sqrt{\frac{\Phi_{0}}{2 \pi H_{c 2}(0)}},
$$

где $\Phi_{0}-$ квант потока. Длина когерентности в грязных сверхпроводниках второго рода ограничивается длиной свободного пробега электронов, которая зависит от пространственной неоднородности сверхпроводящей фазы [22]. Таким образом, близость критических полей в объемном сплаве $\mathrm{Ga}-\mathrm{In}-\mathrm{Sn}$ и в исследуемом нанокомпозите говорит об одинаковой степени неоднородности фазы $\mathrm{In}_{3} \mathrm{Sn}$ в обоих случаях.

Участки с положительной кривизной на зависимости $H_{c 2}(T)$ допускают интерпретацию в рамках модели, рассмотренной в работе [23]. Модель анализирует совокупность нерегулярных сверхпроводящих и несверхпроводящих слоев, связанных джозефсоновским взаимодействием. Эффект близости обуславливает появление ненулевого параметра порядка в несверхпроводящих слоях. Пространственное изменение параметра порядка приводит к положительной кривизне критической линии близко к $T_{c}$. При возрастании магнитного поля параметр порядка в несверхпроводящих слоях сильно уменьшается из-за уменьшения длины когерентности и эффект близости перестает играть заметную роль. На рис. 2 показана аппроксимация участков положительной кривизны на критических зависимостях $H_{c 2}(T)$ с помощью формулы (13) из [23].

На рис. 3 показаны температурные зависимости мнимой части восприимчивости, полученные на частоте $5.01 \mathrm{kHz}$ при различных амплитудах $H_{a c}$ переменного поля в нулевом поле смещения. Видно, что положения пиков, соответствующих обоим сверхпроводящим переходам, существенно сдвигаются к низким температурам 


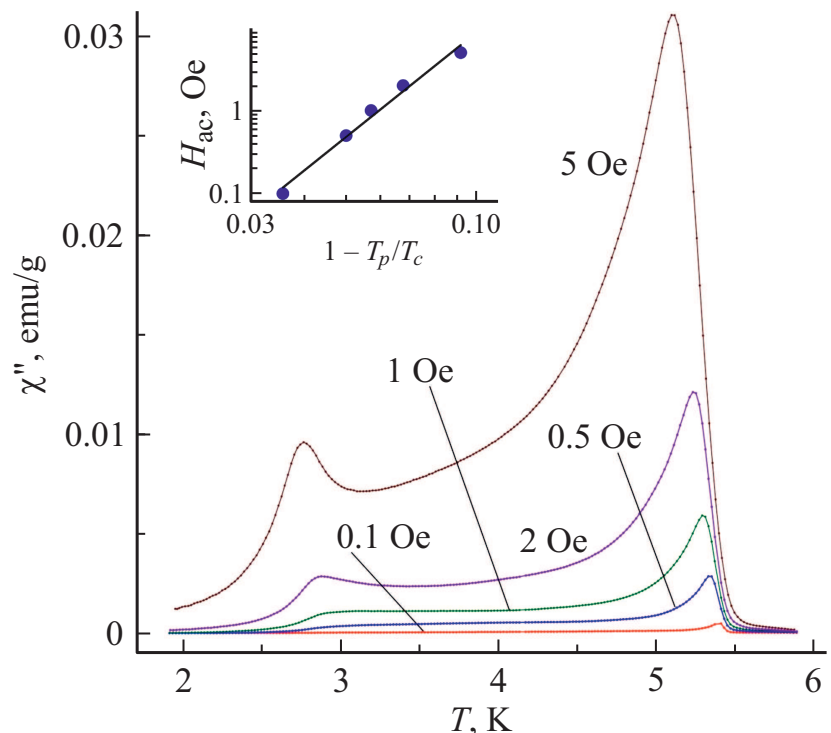

Рис. 3. Мнимая часть восприимчивости, измеренная на частоте $5.01 \mathrm{kHz}$ для амплитуды переменного поля 0.1, 0.5, 1,2 и 5 Ое. На вставке показана зависимость амплитуды переменного поля $H_{a c}$ от $1-T_{p} / T_{c}$, полученная в нулевом поле смещения.

при увеличении амплитуды. На вставке к рис. 3 показано, что связь между температурой максимума восприимчивости $T_{p}$ и $H_{a c}$ для первого сверхпроводящего перехода подчиняется степенному закону

$$
H_{a c} \propto\left(1-\frac{T_{p}}{T_{c}}\right)^{\beta} .
$$

Показатель $\beta$ равен 4.2. Наряду с сильной амплитудной зависимостью положения пиков исследования, проведенные на различных частотах переменного поля, выявили слабое регулярное смещение пиков мнимой части восприимчивости от частоты. Слабое изменение $T_{p}$ с изменением частоты свидетельствует о слабой зависимости критического тока от электрического поля, а также о пропорциональности критического тока амплитуде $H_{a c}$ при температуре $T_{p}$ [24-26]. Таким образом, связь между критическим током и $T_{p}$ дается выражением, аналогичным (3). Как показано в работах [9,27,28], модель гранульных сверхпроводников, в которой гранулы связаны сильными или слабыми джозефсоновскими связями, применима к нанокомпозитам на основе мезопористых твердых матриц с металлическими включениями. При этом гранулы состоят из нескольких соседних пор, соединенных заполненными каналами [6,28,29]. Согласно [30] показатель в формуле (3) равен 3/2 для случая сильно связанных гранул и снижается для случая слабых связей. Для исследуемого нами нанокомпозита показатель значительно больше, что требует дополнительного теоретического анализа. Аналогичный результат был получен для пористого стекла, заполненного сплавом $\mathrm{Ga}-\mathrm{Ag}$ [13]. Для второго сверхпроводящего перехода

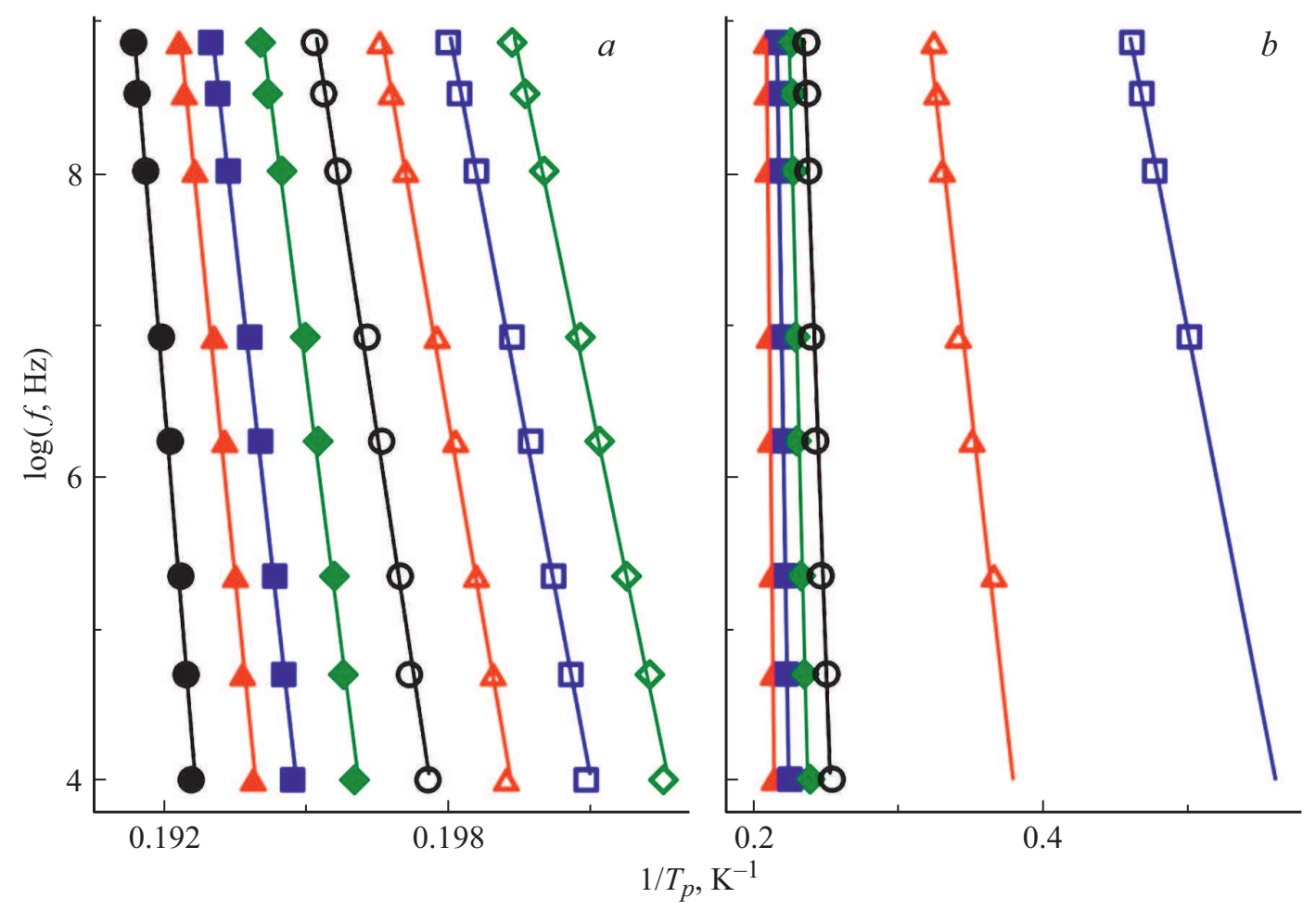

Рис. 4. Аррениусовские графики для первого сверхпроводящего перехода, полученные в полях смещения (слева направо) 10, 50, $100,200,300,500,750$ Ое и $1 \mathrm{kOe}(a)$ и $3,5,7.5,10,20,25 \mathrm{kOe}(b)$. Прямые линии - линейные зависимости. 
имеющихся данных недостаточно для аппроксимации связи между $T_{p}$ и $H_{a c}$.

Сильное влияние на величину $T_{p}$ амплитуды переменного поля и слабая зависимость $T_{p}$ от частоты указывают на термоактивационную природу движения сверхпроводящих вихрей [22,31-33]. Смещения пиков достаточно существенны для того, чтобы построить аррениусовские графики. Эти графики для амплитуды переменного поля 2 Ое представлены на рис. 4 для первого перехода и на рис. 5 для второго перехода. Рис. 4 и 5 демонстрируют линейные зависимости логарифма частоты от обратной температуры максимальных значений мнимой части восприимчивости для обоих сверхпроводящих переходов, что дает возможность рассчитать активационные барьеры $U_{a}$. Величины активационных барьеров для различных смещающих полей представлены на рис. 6 .

Для первого и второго сверхпроводящих переходов на зависимостях активационных барьеров от смещающего поля можно выделить два участка. На обоих участках активационные барьеры изменяются с изменением поля, следуя степенному закону $U_{a} \propto H^{-\alpha}$. В полях до $3 \mathrm{kOe}$ показатель степени $\alpha$ значительно меньше единицы, отражая слабую зависимость $U_{a}$ от поля $(\alpha=0.21$ для первого перехода и $\alpha=0.20$ для второго перехода). Выше $5 \mathrm{kOе}$ зависимость активационных барьеров от поля значительно усиливается и показатель увеличивается до 1.56 и 0.96 для первого и второго переходов соот-

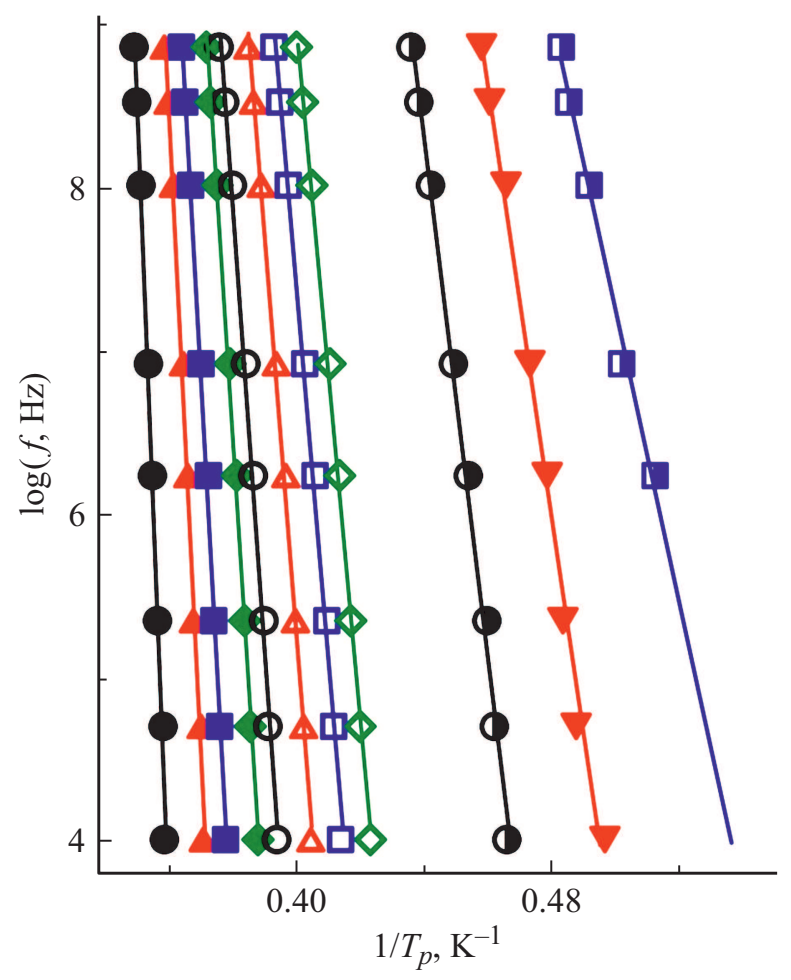

Рис. 5. Аррениусовские графики для второго сверхпроводящего перехода, полученные в полях смещения (слева направо) $10,50,100,200,300,500,750$ Ое и $1,3,5,7.5$ kOe. Прямые линии - линейные зависимости.

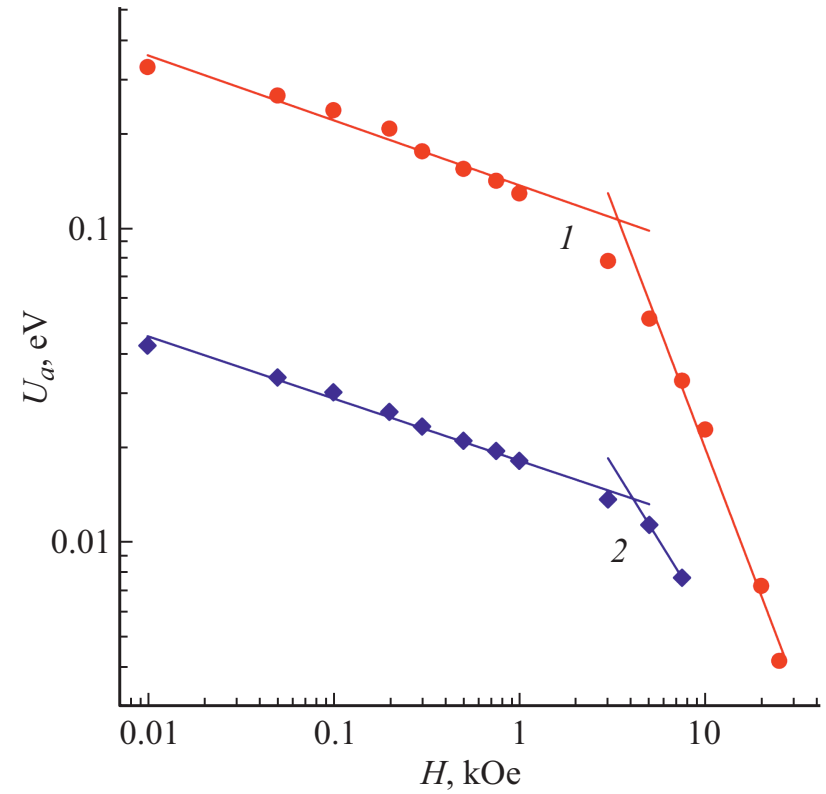

Рис. 6. Зависимость активационных барьеров от поля смещения для первого (1) и второго (2) сверхпроводящих переходов. Прямые линии - степенные зависимости.

ветственно. Изломы на полевой зависимости активационных барьеров наблюдались для различных сверхпроводников второго рода, например, для нетрадиционных сверхпроводников (см. [34-36] и ссылки в этих работах), а также для нанокомпозитов с включениями ряда чистых металлов и сплава галлия с серебром $[5,11,13]$. Обнаруженные изломы трактовались на основе различных моделей, в частности, как проявление фазового перехода в системе вихрей. Для исследуемого композита трансформация в системе вихрей при увеличении поля может быть связана с перераспределением вихрей в пространстве между гранулами и внутри гранул [11]. В малых полях нанокомпозит ведет себя как грязный сверхпроводник второго рода. Вихри закрепляются на неоднородностях между гранулами. При движении к другим центрам пиннинга вихри преодолевают высокие потенциальные барьеры. Усиление поля приводит к постепенному разрыву связей между сверхпроводящими гранулами и переходу межгранульных областей в нормальное состояние. Подвижность вихрей ограничивается размерами гранул. Активационные барьеры уменьшаются из-за большей однородности гранул, что дает излом на полевой зависимости $U_{a}$. В работах $[11,13]$ поля, при которых наблюдался излом, были близки к полям кроссовера от положительной к отрицательной кривизне критической линии. Однако в случае исследуемого в настоящей работе нанокомпозита с тройным сплавом $\mathrm{Ga}-\mathrm{In}-\mathrm{Sn}$ магнитные поля, соответствующие излому и кроссоверу, не коррелируют друг с другом. В рамках модели [23] поле кроссовера определяется полевой зависимостью эффекта близости, на которую влияет морфология нанокомпозита. Таким образом, сле- 
дует предположить, что морфология композита пористое стекло/Ga- In- Sn отличается от морфологии нанокомпозитов, исследованных в работах $[11,13]$.

\section{4. Заключение}

Исследования $a c$ восприимчивости для нанокомпозита пористое стекло/эвтектический сплав $\mathrm{Ga}-\mathrm{In}-\mathrm{Sn}$ выявили двухступенчатый сверхпроводящий переход с характерными температурами 5.6 и $3.1 \mathrm{~K}$. Эти температуры предположительно связаны с формированием в порах интерметаллического соединения $\operatorname{In}_{3} \mathrm{Sn}$ и твердого раствора индия с небольшим количеством галлия и олова. Зависимости верхнего критического поля от температуры имеют участки с положительной кривизной и допускают интерпретацию на основе модели, учитывающей эффект близости. Сильная амплитудная и слабая частотная зависимости мнимой части восприимчивости указывают на активационную природу движения вихрей. Рассчитанные активационные барьеры изменяются с изменением полей смещения по степенному закону. При этом показатель степени значительно возрастает в сильных полях.

\section{Финансирование работы}

Исследования финансировались РНФ (грант 21-7220038). Измерения проводились на оборудовании Ресурсного центра „Центр диагностики функциональных материалов для медицины, фармакологии и наноэлектроники“ Научного парка Санкт-Петербургского государственного университета.

\section{Конфликт интересов}

Авторы заявляют, что у них нет конфликта интересов.

\section{Список литературы}

[1] The Oxford handbook of small superconductors / Ed. A.V. Narlikar. Oxford University Press, Oxford (2017).

[2] Superconductors at the nanoscale. From basic research to applications / Ed. R. Wördenweber, V. Moshchalkov, S. Bending, F. Tafuri. eBook De Gruyter (2017).

[3] Y. Kumzerov, S. Vakhrushev. In: Encyclopedia of Nanoscience and Nanotechnology / Ed. H.S. Nalwa. American Scientific Publishers (2004). V. 7. P. 811.

[4] M. Salvato, R. Baghdadi, C. Cirillo, S.L. Prischepa, A.L. Dolgiy, V.P. Bondarenko, F. Lombardi, C. Attanasio. Nanotechnology 28, 465301 (2017).

[5] M.K. Lee, E.V. Charnaya, C. Tien, L.J. Chang, Y.A. Kumzerov, J. Appl. Phys. 113, 113903 (2013).

[6] E.V. Charnaya, C. Tien, K.J. Lin, Y.A. Kumzerov. Phys. Rev. B 58, 11089 (1998).

[7] E.V. Charnaya, C. Tien, M.K. Lee, Y.A. Kumzerov. J. Phys.: Conden. Matter 21, 455304 (2009).

[8] M.K. Lee, C. Tien, E.V. Charnaya, H.-S. Sheu, Y.A. Kumzerov. Phys. Lett. A 374, 1570 (2010).
[9] Y.S. Ciou, M.K. Lee, E.V. Charnaya, C. Tien, L.J. Chang, Y.A. Kumzerov, M.I. Samoylovich. Supercond. Sci. Technol. 26, 055009 (2013).

[10] C. Tien, E.V. Charnaya, D.Y. Xing, A.L. Pirozerskii, Y.A. Kumzerov, Y.S. Ciou, M.K. Lee. Phys. Rev. B 83, 014502 (2011).

[11] M.K. Lee, E.V. Charnaya, S. Mühlbauer, U. Jeng, L.J. Chang, Y.A. Kumzerov. Sci. Rep. 11, 4807 (2021).

[12] D.Yu. Nefedov, E.V. Charnaya, A.V. Uskov, A.O. Antonenko, D.Yu. Podorozhkin, J. Haase, Y.A. Kumzerov, A.V. Fokin. Appl. Magn. Res. (2021). Published online. DOI 10.1007/s00723-021-01385-4.

[13] E.V. Shevchenko, E.V. Charnaya, M.K. Lee, L.J. Chang, M.V. Likholetova, I.E. Lezova, Y.A. Kumzerov, A.V. Fokin. Physica C 574, 1353666 (2020).

[14] N. Ochirkhuyag, R. Matsuda, Z. Song, F. Nakamura, T. Endo, H. Ota. Nanoscale 13, 2113 (2021).

[15] G. Bo, L. Ren, X. Xu, Y. Du, S. Dou. Adv. Phys. X 3, 1446359 (2018).

[16] T. Mochiku, M. Tachiki, S. Ooi, Y. Matsushita. Physica C 263, 33 (2019).

[17] L. Ren, J. Zhuang, G. Casillas, H. Feng, Y. Liu, X. Xu, Y. Liu, J. Chen, Y. Du, L. Jiang, S.X. Dou. Adv. Func. Mater. 26, 8111 (2016).

[18] Y. Plevachuk, V. Sklyarchuk, S. Eckert, G. Gerbeth, R. Novakovic. J. Chem. Eng. Data 59, 757 (2014).

[19] Y. Shu, T. Ando, Q. Yin, G. Zhou, Z. Gu. Nanoscale 9, 12398 (2017).

[20] M.F. Merriam, M. Von Herzeni. Phys. Rev. 131, 637 (1963).

[21] N.R. Werthamer, E. Helfand, P.C. Hohenberg. Phys. Rev. 147, 295 (1966).

[22] М. Тинкхам. Введение в сверхпроводимость. Атомиздат, M. (1980). [Tinkham M. Introduction to Superconductivity. 2 nd. ed. Dover Publications (2004)].

[23] S. Theodorakis, Z. Teanovic. Phys. Rev. B 40, 6659 (1989).

[24] E.H. Brandt. Phys. Rev. B 55, 14513 (1997).

[25] F. Gömöry. Supercond. Sci. Technol. 10, 523 (1997).

[26] J.R. Clem, A. Sanchez. Phys. Rev. B 50, 9355 (1994).

[27] E.V. Charnaya, C. Tien, K.J. Lin, C.-S. Wur, Y.A. Kumzerov. Phys. Rev. B 58, 467 (1998).

[28] E.V. Charnaya, C. Tien, C S. Wur, Y.A. Kumzerov. Physica C 269, 313 (1996).

[29] E.V. Charnaya, C. Tien, M.K. Lee, Y.A. Kumzerov. In: Indium: Properties, Technological Applications and Health Issues (2013). P. 1.

[30] J.R. Clem, B. Bumble, S.I. Raider, W.J. Gallagher, Y.C. Shih. Phys. Rev. B 35, 6637 (1987).

[31] T.T.M. Palstra, B. Batlogg, R.B. van Dover, L.F. Schneemeyer, J.V. Waszczak. Phys. Rev. B 41, 6621 (1990).

[32] P.W. Anderson, Y.B. Kim. Rev. Mod. Phys. 36, 39 (1964).

[33] G. Prando, P. Carretta, R. De Renzi, S. Sanna, A. Palenzona, M. Putti, M. Tropeano. Phys. Rev. B 83, 174514 (2011).

[34] G. Prando, P. Carretta, R. De Renzi, S. Sanna, H.-J. Grafe, S. Wurmehl, B. Büchner. Phys. Rev. B 85, 144522 (2012).

[35] S.R. Ghorbani, X.L. Wang, M. Shabazi, S.X. Dou, K.Y. Choi, C.T. Lin. Appl. Phys. Lett. 100, 072603 (2012).

[36] H. Lei, R. Hu, C. Petrovic. Phys. Rev. B 84, 014520 (2011).

Редактор К.В. Емщев 